\title{
Espondiloartropatía destructiva no infecciosa en un paciente en hemodiálisis
}

\section{Destructive noninfectious spondyloarthropathy in a hemodialysis patient}

\author{
Arbey Aristizábal-Alzate, Kelly Johanna Betancur-Salazar, John Fredy Nieto-Rios, \\ Catalina Ocampo-Kohn, Dahyana Cadavid-Aluure, Martín Ochoa-Escudero, \\ Gustavo Zuluaga-Valencia • Medellín (Colombia) \\ Lina María Serna-Higuita • Tuebingen (Germany)
}

DOI: https://doi.org/10.36104/amc.2022.2193

\begin{abstract}
Resumen
La espondiloartropatía destructiva es una patología osteoarticular presente en algunos pacientes con enfermedad crónica que puede afectar varios niveles de la columna vertebral y puede ser asintomática, generar dolor o causar complicaciones que ponen en peligro la integridad de la médula espinal y/o la vida. Presentamos el caso de un hombre de 70 años con enfermedad renal crónica terminal en hemodiálisis quien consultó por dolor dorsal y paraplejia, en quien se diagnosticó espondiloartropatía destructiva no infecciosa por imágenes y estudio histopatológico. Este caso nos muestra la importancia de pensar en esta patología y la necesidad de un enfoque multidisciplinario en el diagnóstico y manejo. (Acta Med Colomb 2022; 47. DOI: https:// doi.org/10.36104/amc.2022.2193).
\end{abstract}

Palabras clave: espondiloartropatía destructiva, enfermedad renal crónica, diálisis, paraplejia.

\begin{abstract}
Destructive spondyloarthropathy is a bone and joint disease which presents in some patients with chronic illnesses and may affect various levels of the spinal column. It may be asymptomatic, cause pain, or produce spinal cord and/or life-endangering complications. We present the case of a 70 -year-old man with end-stage renal disease on hemodialysis who consulted due to back pain and paraplegia. He was diagnosed with destructive noninfectious spondyloarthropathy through imaging and histopathological studies. This case shows us the importance of considering this disease and the need for a multidisciplinary approach in its diagnosis and management. (Acta Med Colomb 2022; 47. DOI: https://doi.org/10.36104/amc.2022.2193).
\end{abstract}

Key words: destructive spondyloarthropathy, chronic kidney disease, dialysis, paraplegia.
Dres. Arbey Aristizábal-Alzate, Dahyana Cadavid-Aljure, Gustavo Zuluaga Valencia: Departamento de Nefrología y Trasplante Renal Hospital Pablo Tobón Uribe; Dra. Kelly Johanna Betancur-Salazar: Departamento de Medicina Interna, Universidad Pontificia Bolivariana; Dres. John Fredy Nieto-Ríos, Catalina OcampoKohn: Departamento de Nefrología y Trasplante Renal Hospital Pablo Tobón Uribe. Departamento de Medicina Interna, Sección Nefrología Universidad de Antioquia; Dr. Martín OchoaEscudero: Departamento de Radiología Hospital Pablo Tobón Uribe. (Medellín Colombia). Dra. Lina María Serna-Higuita: Eberhard Karls University, Institute for Clinical Epidemiology und Applied Biometrics. Tuebingen (Germany). Correspondencia: Dr. John Fredy Nieto-Ríos. (Medellín Colombia).

E-Mail: johnfredynieto@gmail.com,linasernahiguita@gmail.com

Recibido: 27/IV/2021 Aceptado: 10/V/2021

\section{Introducción}

Los pacientes con enfermedad renal crónica terminal (ERCT) pueden presentar una amplia gama de alteraciones osteoarticulares, incluyendo osteodistrofia renal, artritis inducida por cristales, necrosis avascular, calcificación de tejidos blandos, amiloidosis e infecciones óseas, entre otras (1). Dentro de estas alteraciones, la espondiloartropatía destructiva (ED) ha sido reconocida como una entidad observada en los pacientes con ERCT, la cual puede llevar a complicaciones graves que ponen en riesgo la funcionalidad y la vida de los pacientes (2). A continuación, presentamos el caso de un paciente en hemodiálisis que consultó por dolor dorsal y paraplejia, en el cual se encontró destrucción de cuerpos vertebrales dorsales secundario a una espondiloartropatía destructiva asociada a su enfermedad renal crónica.

\section{Presentación del caso}

Hombre de 70 años con antecedentes de nefropatía diabética y ERCT de seis años de evolución en hemodiálisis. Consultó por 15 días de evolución de intenso dolor en región dorsal, parestesias de miembros inferiores e imposibilidad para la marcha. Al examen físico se observó un paciente con paraplejia, con intenso dolor al palpar vértebras dorsales T5 y T6. Inicialmente se sospechó una espondilodiscitis infecciosa. Los estudios de laboratorios reportaron un valor de creatinina de $6.39 \mathrm{mg} / \mathrm{dL}$, PCR $0.43 \mathrm{mg} / \mathrm{dL}, \mathrm{Hb} 10.8 \mathrm{gr} / \mathrm{L}$, 
leucocitos 7500, plaquetas 152000 , VSG 23 y hemocultivos negativos. La radiografía de columna mostró colapso en cuerpo vertebral $\mathrm{T} 5$ con acuñamiento anterior y pérdida de altura en un $50 \%$, además pérdida del espacio intervertebral T5-T6 (Figura 1). La tomografía computarizada describió T5-T6 con marcada irregularidad y esclerosis del espacio intervertebral con destrucción de las láminas terminales. La resonancia mostró alteración difusa en la intensidad de cuerpos vertebrales, con hipointensidad en STIR, pérdida de la altura e irregularidad marcada de los platillos terminales y del disco T5-T6, edema de los cuerpos vertebrales y retropulsión del muro posterior hacia el canal medular que generó un canal estrecho de $3.5 \mathrm{~mm}$ con compresión del cordón medular (Figuras 2 y 3). Fue llevado a cirugía donde se identificó lesión en T5-T6, se realizó disección paravertebral hasta láminas y apófisis transversas, laminectomía T5 y T6. Se estabilizó la columna y se tomaron muestras múltiples para cultivo y patología. Se iniciaron antibióticos (piperacilina tazobactam y vancomicina) los cuales fueron suspendidos ya que todos los cultivos de muestra de cirugía (hueso y disco) fueron negativos. El estudio histopatológico reportó la presencia de fragmentos de tejido óseo, cartilaginoso y fibroconectivo con cambios

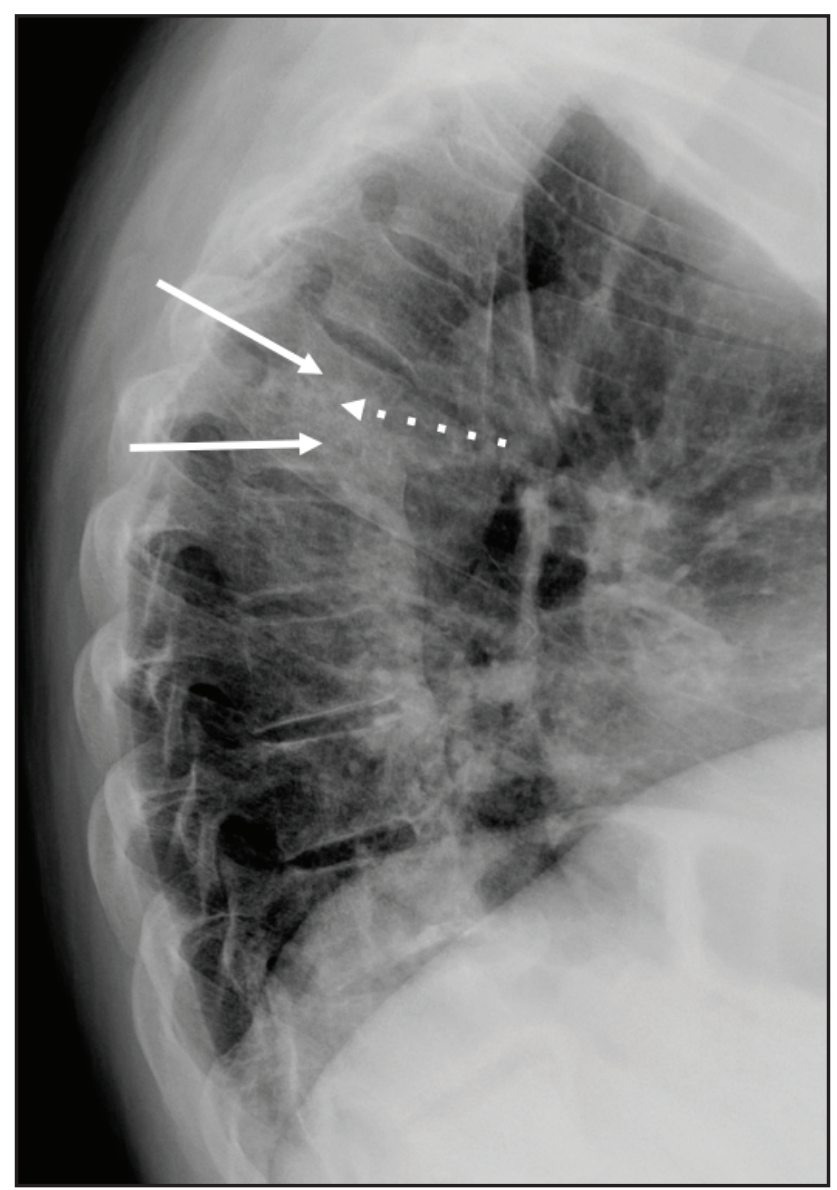

Figura 1. Proyección lateral de radiografía de columna dorsal en la cual se observa colapso parcial de los cuerpos vertebrales de T5 y T6 (flechas continuas), acompañado de pérdida del espacio intervertebral y esclerosis de las láminas terminales en T5-T6 (flecha punteada). degenerativos, inflamación crónica y cambios reactivos, tinción de rojo Congo y cristal violeta fue negativa para amiloide. Se descartó infección o malignidad y se inició estudio para trastorno del metabolismo mineral óseo. Los paraclínicos mostraron hiperparatiroidismo grave (PTH $1291 \mathrm{pg} / \mathrm{mL}$ ), hiperfosfatemia (fósforo $12 \mathrm{mg} / \mathrm{dL}$ ), hipercalcemia (calcio $10.7 \mathrm{mg} / \mathrm{dL}$ ), elevación de B2 microglobulina $(18.3 \mathrm{mg} / \mathrm{L})$, resultados que apoyaron el diagnóstico de ED secundaria a hiperparatiroidismo. Por tal motivo se realizó paratiroidectomía (3/4 paratiroides) con evolución adecuada. La patología reportó tejido paratiroideo con hiperplasia marcada. Última evaluación con normalización del perfil calcio-fósforo, con paraplejia como secuela y dolor crónico y sin otras complicaciones.

\section{Discusión}

Se reporta el caso de un paciente con ERCT en hemodiálisis que presentó paraplejia por compresión de la médula espinal dorsal secundario a ED, complicación rara y posiblemente subdiagnóstica en los pacientes con ERCT. Esta enfermedad se presenta principalmente en la porción más baja de la columna cervical, aunque también puede afectar la unión craneocervical como en el caso presentado (3-5), lo

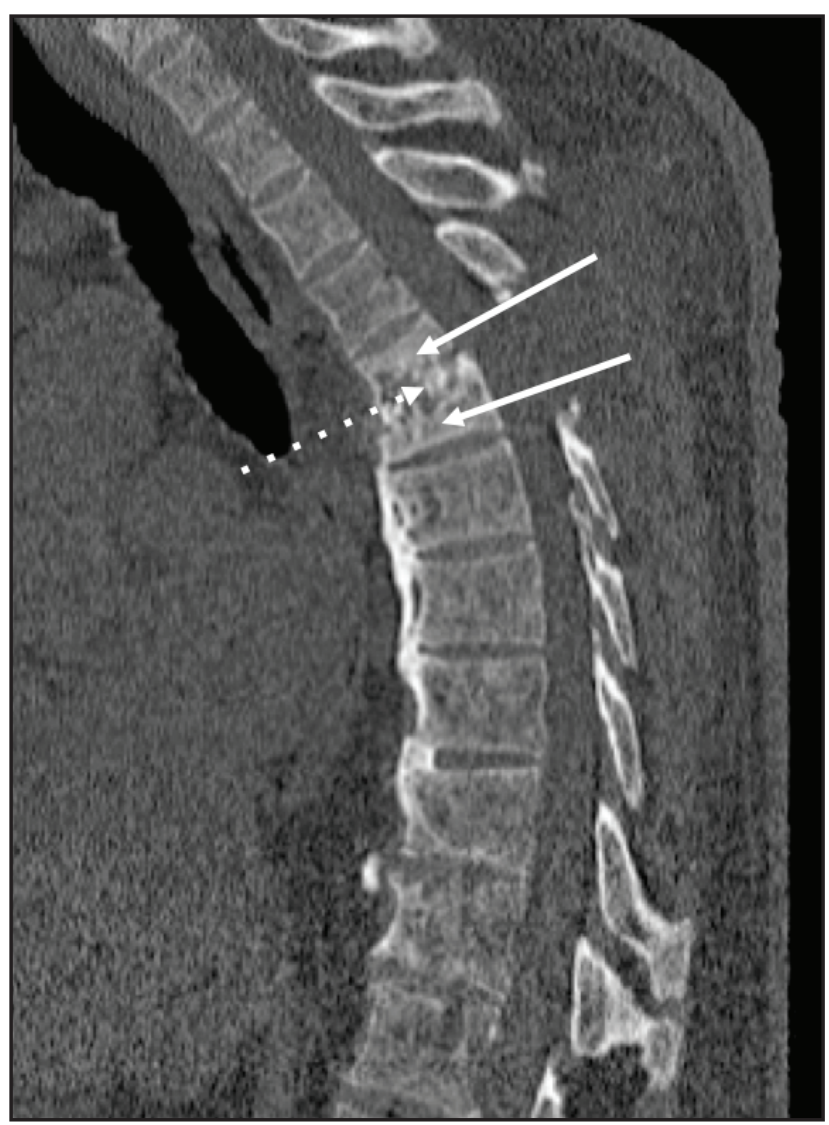

Figura 2. Reconstrucción sagital de tomografía de columna dorsal sin medio de contraste en ventana para hueso, en la cual se aprecia colapso parcial de los cuerpos vertebrales de T5-T6 (flechas continuas) y pérdida del espacio intervertebral T5-T6 acompañada de irregularidad y esclerosis de las láminas terminales (flecha punteada). Estos hallazgos sugerían espondilodiscitis. 


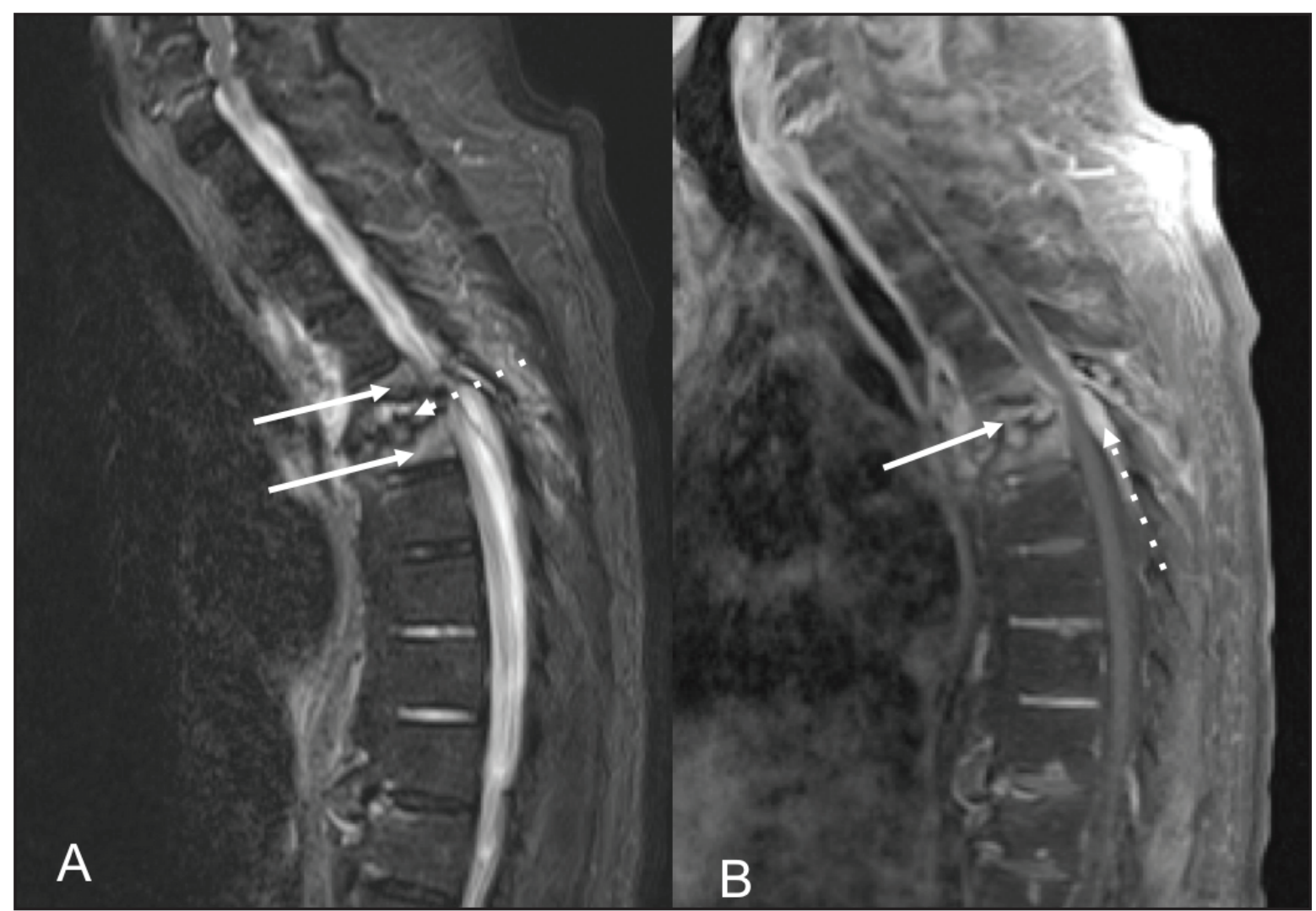

Figura 3. (A) Secuencia STIR sagital en la cual se observa colapso parcial e hiperintensidad de los cuerpos vertebrales de T5 y T6 (flechas continuas). Además, se aprecia pérdida de la altura del espacio intervertebral T5-T6 con irregularidad de las láminas terminales e hiperintensidad del disco intervertebral (flecha punteada). (B) Secuencia sagital potenciada en $T 1$ con medio de contraste y supresión de grasa en la cual se identifica realce del espacio y del disco intervertebral (flecha continua) y engrosamiento y realce del espacio epidural (flecha punteada). Los hallazgos fueron interpretados como espondilodiscitis con extensión al espacio epidural.

cual puede llevar a la muerte por compresión de estructuras del tallo cerebral (6-8).

La patogénesis y fisiopatología de la ED no se conoce completamente, se ha relacionado con la duración de la insuficiencia renal, duración de la diálisis y bioincompatibilidad de las membranas de diálisis. El hiperparatiroidismo grave que lleva a resorción del hueso subcondral se ha sugerido como un factor causal en las anormalidades vertebrales (9). Además hay evidencia que indica que los depósitos de amiloide, compuestos de $\beta 2$-microglobulina que pueden contribuir al desarrollo de cambios destructivos espinales en pacientes con diálisis crónica $(10,11)$. En este reporte se encontró hiperparatiroidismo severo con PTH muy elevada, hiperfosfatemia e hipercalcemia como factor asociado a la destrucción ósea evidenciada.

La prevalencia de la ED en paciente con ERCT oscila entre 5 y $25.3 \%(3,5,12,13)$. El tiempo promedio entre el inicio de la terapia de reemplazo renal y la presencia de ED es de 9.6 años (14). Sin embargo, algunos estudios han descrito esta complicación en las fases iniciales de la enfermedad renal (15). Además hay casos reportados de ED asociadas con insuficiencia renal crónica en ausencia de terapia dialítica $(16,17)$. La prevalencia reportada en diferentes series de casos oscila entre 12 y $34 \%$ en pacientes con hemodiálisis y 10-39\% en pacientes en diálisis peritoneal $(15,18,19)$. Sin embargo, es difícil comparar su prevalencia con las dos modalidades de diálisis debido a que es poco frecuente que los pacientes en diálisis peritoneal permanezcan en esta terapia por más de una década.

Diferentes estudios imagenológicos se han usado para evaluar los pacientes con sospecha de espondiloartropatía. La radiografía por su alta resolución espacial y bajo costo se considera el examen de elección para la evaluación inicial de las anormalidades espinales. Las características radiográficas de la ED consisten en el estrechamiento u obliteración de los espacios de los discos intervertebrales, erosión o resorción del hueso subcondral en los platillos terminales superior e inferior de los cuerpos vertebrales, con o sin lesiones quísticas, esclerosis del hueso subcondral y mínima osteofitosis $(12,14,20,21)$. Pueden observarse además la afectación de niveles espinales únicos o múltiples y las lesiones pueden involucrar cuerpos vertebrales contiguos o distantes $(3,22)$. Las anormalidades vertebrales tempranas pueden simular las de espondilitis anquilosante temprana $(14,22)$. Con la progresión de la enfermedad las lesiones destructivas pueden simular infección (espondilodiscitis) con estrechamiento del espacio del disco e irregularidad y esclerosis del hueso subcondral en los cuerpos vertebrales 
adyacentes. En estadios avanzados de la enfermedad, el cuerpo vertebral se fractura y colapsa, y se puede observar subluxación o espondilolistesis $(2,12,20)$.

La tomografía computarizada es el mejor método para detectar pequeñas áreas de osteólisis en el hueso cortical, en la valoración de la distribución y extensión de los cambios destructivos $(21,23)$ y para descartar la presencia de abscesos. Entre los hallazgos tomográficos que sugieren espondilitis infecciosa son el estrechamiento del espacio de los discos intervertebrales, irregularidad de los platillos terminales vertebrales adyacentes y una masa de tejido blando prevertebral $(24,25)$. La tomografía puede ser útil además para demostrar subluxación vertebral y permitir la evaluación de compresión espinal (26).

La gammagrafía es altamente sensible para detectar presencia de infección ósea aguda, aunque no es específica para valorar la naturaleza exacta y extensión de la infección (27). La gammagrafía con tecnecio-99 ha sido usada para visualizar depósito de amiloide periarticular, aunque no diferencia entre artropatía inducida por amiloide y artropatías de origen diferente (27). El uso de gammagrafía con yodo-131 o indio-111 radiomarcado con la proteína B2 microglobulina precursora de amiloide puede generar resultados más confiables $(28,29)$. En cuanto a la resonancia magnética, se resalta que es altamente sensible en detectar la presencia y extensión de infección en huesos, articulaciones y tejido blando $(30,31)$.

Entre los diagnósticos diferenciales de la ED se encuentran las lesiones metastásicas, mieloma múltiple, osificación del ligamento longitudinal posterior y espondilosis cervical (32). En la enfermedad metastásica las lesiones son más difusas y menos circunscritas. El mieloma múltiple puede ser diferenciado de espondiloartropatía secundaria a amiloidosis relacionada con la diálisis por electroforesis de proteínas en suero y orina. Una gammagrafía ósea puede ser usada para detectar otras lesiones de mieloma y para esto también puede ser útil la resonancia magnética. En caso de sospecha de espondilodiscitis, como en el caso reportado, la literatura también ha descrito la necesidad de efectuar biopsia de las lesiones que puede ser por aspiración con aguja o con cirugía, siendo la cirugía la que aporta información más consistente; sin embargo, tiene mayor morbilidad que la aspiración que es reconocida como un método seguro (13).

El abordaje terapéutico de la ED se basa en el control de los síntomas, la estabilización ortopédica o quirúrgica de la columna y el manejo de la causa subyacente. En pacientes con compromiso medular, es prioritaria la descompresión quirúrgica y estabilización de la columna; evaluando siempre el riesgo/beneficio dado el alto riesgo quirúrgico de los pacientes con ERCT en hemodiálisis (33). Los problemas de la cirugía no sólo tienen que ver con las complicaciones del sitio quirúrgico como la susceptibilidad a infección bacteriana y severa fragilidad del hueso, sino también con condiciones generales como anemia, alteraciones electrolíticas y de fluidos y control de la presión sanguínea, se recomienda en esos pacientes con tratamiento multidisciplinario y así prevenir las complicaciones intra y posoperatorias (34).

Dada la correlación fisiopatológica de la ED con el depósito amiloide, se ha postulado el cambio de membranas de alto flujo como estrategia para disminuir la acumulación de beta-2-microglobulina, pero esto no ha sido validado. En aquellos pacientes en quienes se demuestra hiperparatiroidismo la paratiroidectomía está indicada, lo que previene complicaciones y lesiones futuras; tal fue el caso del paciente reportado que requirió esta cirugía por hiperparatiroidismo grave. Se debe considerar además el trasplante renal como estrategia de tratamiento efectiva, ya que esto resuelve el problema del depósito amiloide y el trastorno en el metabolismo mineral óseo que hacen parte de la fisiopatología de la enfermedad (1).

En conclusión, la ED es una complicación de la enfermedad renal crónica avanzada con presentación clínica variable y su prevalencia es de $10-15 \%$ en población en diálisis. El tiempo en diálisis y la edad avanzada son los predictores más fuertes para su desarrollo. Se diagnostica con base en hallazgos imagenológicos de grave estrechamiento del espacio discal y erosiones vertebrales adyacentes sin osteofitosis significativa; sin embargo, cuando el diagnóstico no es claro, el estudio histopatológico es fundamental para descartar infección y malignidad. El mayor desafío clínico es distinguirla de un proceso infeccioso. El objetivo del manejo es mejorar los síntomas y prevenir déficits neurológicos. Como actualmente los pacientes con enfermedad renal crónica en estadio terminal viven más, se espera que esta complicación sea observada con mayor frecuencia.

\section{Referencias}

1. Pak F, Lash J. Destructive spondyloarthropathy: an overview. Int J Artif Organs. 1999;22(8):531-5

2. Kuntz D, Naveau B, Bardin T, Drueke T, Treves R, Dryll A. Destructive spondylarthropathy in hemodialyzed patients. A new syndrome. Arthritis Rheum. 1984;27(4):369-75.

3. Leone A, Sundaram M, Cerase A, Magnavita N, Tazza L, Marano P. Destructive spondyloarthropathy of the cervical spine in long-term hemodialyzed patients: a five-year clinical radiological prospective study. Skeletal Radiol.2001;30(8):43141.

4. Theodorou DJ, Theodorou SJ, Resnick D. Imaging in dialysis spondyloarthropathy. Semin Dial. 2002;15(4):290-6.

5. Maruyama H, Gejyo F, Arakawa M. Clinical studies of destructive spondyloarthropathy in long-term hemodialysis patients. Nephron. 1992;61(1):37-44.

6. Deforges-Lasseur C, Combe C, Cernier A, Vital JM, Aparicio M. Destructive spondyloarthropathy presenting with progressive paraplegia in a dialysis patient. Recovery after surgical spinal cord decompression and parathyroidectomy. Nephrol Dial Transplant. 1993;8(2):180-4.

7. Mikawa Y, Yamaoka T, Watanabe R. Compression of the spinal cord due to destructive spondyloarthropathy of the atlanto-axial joints. J Bone Joint Surg Am. 1996;78(12):1911-4

8. Allard JC,Artze ME, Porter G, Ghandur-Mnaymneh L, de Velasco R, Perez GO. Fatal destructive cervical spondyloarthropathy in two patients on long-term dialysis. Am J Kidney Dis. 1992;19(1):81-5.

9. McCarthy JT, Dahlberg PJ, Kriegshauser JS, Valente RM, Swee RG, O’Duffy JD, et al. Erosive spondyloarthropathy in long-term dialysis patients: relationship to severe hyperparathyroidism. Mayo Clin Proc. 1988;63(5):446-52.

10. Ohashi K, Hara M, Kawai R, Ogura Y, Honda K, Nihei H, et al. Cervical discs 
are most susceptible to beta 2-microglobulin amyloid deposition in the vertebral column. Kidney Int. 1992;41(6):1646-52.

11. Davidson GS, Montanera WJ, Fleming JF, Gentili F. Amyloid destructive spondyloarthropathy causing cord compression: related to chronic renal failure and dialysis. Neurosurgery. 1993;33(3):519-22.

12. Kerr R, Bjorkengren A, Bielecki DK, Resnick D, Feinstein EI. Destructive spondyloarthropathy in hemodialysis patients. Report of four cases and prospective study. Skeletal Radiol. 1988;17(3):176-80.

13. Bindi P, Chanard J. Destructive spondyloarthropathy in dialysis patients: an overview. Nephron. 1990;55(2):104-9.

14. Naidich JB, Mossey RT, McHeffey-Atkinson B, Karmel MI, Bluestone PA, Mailloux LU, et al. Spondyloarthropathy from long-term hemodialysis. Radiology. 1988;167(3):761-4.

15. Menard HA, Langevin S, Levesque RY. Destructive spondyloarthropathy in short term chronic ambulatory peritoneal dialysis and hemodialysis. J Rheumatol. 1988;15(4):644-7.

16. Alcalay M, Goupy MC, Azais I, Bontoux D. Hemodialysis is not essential for the development of destructive spondylarthropathy in patients with chronic renal failure. Arthritis Rheum. 1987;30(10):1182-6.

17. Moriniere P, Marie A, el Esper N, Fardellone P, Deramond H, Remond A, et al. Destructive spondyloarthropathy with beta 2-microglobulin amyloid deposits in a uremic patient before chronic hemodialysis. Nephron. 1991;59(4):654-7.

18. Gonzalez T, Cruz A, Balsa A, Jimenez C, Selgas R, Miguel JL, et al. Erosive azotemic osteoarthropathy of the hands in chronic ambulatory peritoneal dialysis and hemodialysis. Clin Exp Rheumatol. 1997;15(4):367-71.

19. Hayami N, Hoshino J, Suwabe T, Sumida K, Mise K, Hamanoue S, et al. Destructive Spondyloarthropathy in Patients on Long-Term Peritoneal Dialysis or Hemodialysis. Ther Apher Dial. 2015;19(4):393-8.

20. Resnick D, Kransdorf M. Parathyroid disorders and renal osteodystrophy. In: Elsevier, editor. Bone and Joint Imaging. 3 ed2005.

21. Kaplan P, Resnick D, Murphey M, Heck L, Phalen J, Egan D, et al. Destructive noninfectious spondyloarthropathy in hemodialysis patients: a report of four cases. Radiology. 1987;162(1 Pt 1):241-4.
22. Sundaram M, Seelig R, Pohl D. Vertebral erosions in patients undergoing maintenance hemodialysis for chronic renal failure. AJR Am J Roentgenol. 1987;149(2):323-7.

23. Rafto SE, Dalinka MK, Schiebler ML, Burk DL, Jr., Kricun ME. Spondyloarthropathy of the cervical spine in long-term hemodialysis. Radiology. 1988;166(1 Pt 1):201-4.

24. Kattapuram SV, Phillips WC, Boyd R. CT in pyogenic osteomyelitis of the spine. AJR Am J Roentgenol. 1983;140(6):1199-201.

25. Golimbu C, Firooznia H, Rafii M. CT of osteomyelitis of the spine. AJR Am J Roentgenol. 1984;142(1):159-63.

26. Orzincolo C, Bedani PL, Scutellari PN, Cardona P, Trotta F, Gilli P. Destructive spondyloarthropathy and radiographic follow-up in hemodialysis patients. Skeletal Radiol. 1990;19(7):483-7.

27. Alazraki N. Radionuclide techniques. In: Resnick D, Kransdorf M, editors. Bone and Joint Imaging 2005.

28. Ketteler M, Koch KM, Floege J. Imaging techniques in the diagnosis of dialysisrelated amyloidosis. Semin Dial. 2001;14(2):90-3.

29. Floege J, Burchert W, Brandis A, Gielow P, Nonnast-Daniel B, Spindler E, et al. Imaging of dialysis-related amyloid (AB-amyloid) deposits with 131I-beta 2-microglobulin. Kidney Int. 1990;38(6):1169-76.

30. McEnery K, Murphy W. Magnetic resonance imaging. In: Resnick D, Kransdorf M, editors. Bone and Joint Imaging 1996. p. 84-93.

31. Modic MT, Feiglin DH, Piraino DW, Boumphrey F, Weinstein MA, Duchesneau PM, et al. Vertebral osteomyelitis: assessment using MR. Radiology. 1985;157(1):157-66.

32. Turnaoglu H, Haberal KM, Unal O, Ozen OI,Agildere AM. Cervical destructive spondyloarthropathy due to the dialysis-related amyloidosis: imaging findings. Ann Clin Anal Med. 2019;10(4):525-8.

33. Kumar A, Leventhal MR, Freedman EL, Coburn J, Delamarter R. Destructive spondyloarthropathy of the cervical spine in patients with chronic renal failure. Spine (Phila Pa 1976). 1997;22(5):573-7; discussion 8.

34. Abumi K, Ito M, Kaneda K. Surgical treatment of cervical destructive spondyloarthropathy (DSA). Spine. 2000;25(22):2899-905. 